\title{
Dissipative Nonlinear Schrödinger Equation for Envelope Solitary Rossby Waves with Dissipation Effect in Stratified Fluids and Its Solution
}

\author{
Yunlong Shi, ${ }^{1,2,3}$ Baoshu Yin,,3 Hongwei Yang, ${ }^{4}$ Dezhou Yang, ${ }^{1,3}$ and Zhenhua Xu ${ }^{1,3}$ \\ ${ }^{1}$ Institute of Oceanology, Chinese Academy of Sciences, Qingdao 266071, China \\ ${ }^{2}$ University of Chinese Academy of Sciences, Beijing 100049, China \\ ${ }^{3}$ Key Laboratory of Ocean Circulation and Wave, Chinese Academy of Sciences, Qingdao 266071, China \\ ${ }^{4}$ College of Mathematics and System Science, Shandong University of Science and Technology, Qingdao 266590, China
}

Correspondence should be addressed to Baoshu Yin; baoshuyin@126.com

Received 3 July 2014; Accepted 9 August 2014; Published 19 August 2014

Academic Editor: Huanhe Dong

Copyright (C) 2014 Yunlong Shi et al. This is an open access article distributed under the Creative Commons Attribution License, which permits unrestricted use, distribution, and reproduction in any medium, provided the original work is properly cited.

We solve the so-called dissipative nonlinear Schrödinger equation by means of multiple scales analysis and perturbation method to describe envelope solitary Rossby waves with dissipation effect in stratified fluids. By analyzing the evolution of amplitude of envelope solitary Rossby waves, it is found that the shear of basic flow, Brunt-Vaisala frequency, and $\beta$ effect are important factors to form the envelope solitary Rossby waves. By employing trial function method, the asymptotic solution of dissipative nonlinear Schrödinger equation is derived. Based on the solution, the effect of dissipation on the evolution of envelope solitary Rossby wave is also discussed. The results show that the dissipation causes a slow decrease of amplitude of envelope solitary Rossby waves and a slow increase of width, while it has no effect on the propagation velocity. That is quite different from the KdV-type solitary waves. It is notable that dissipation has certain influence on the carrier frequency.

\section{Introduction}

In the last decades, Rossby waves theory has attracted significant attention for it can testify some of the prime events of geophysical fluid flows. Long [1] and Benney [2] studied long waves in barotropic fluid and obtained a KdV equation, which is a famous soliton wave equation and also can be used to describe internal tide [3-5]. Domaracki and Loesh [6] and Ripa [7] discussed the interaction of two envelope solitary waves, but they did not consider the effect of basic flow on the equatorial envelope solitary waves. Yang et al. [8] studied the solitary Rossby waves induced by linear topography in barotropic fluids with a shear flow. Tan [9] studied the effects of forcing and dissipation on the collision interaction of two envelope solitary waves. Song and Yang [10] derived an inhomogeneous $\mathrm{mKdV}$ equation including topographic forcing by employing the perturbation method and stretching transforms of time and space in stratified fluids. Song and Yang [11] obtained an inhomogeneous BDO-Burgers equation including underlying surface, slowly changing underlying surface, and turbulent dissipation. Maslowe and Redekopp [12] examined nonlinear long waves in stratified flows and discussed the effect of shear on long waves in stratified flows. Luo $[13,14]$ derived a higher order nonlinear Schrödinger equation using a perturbation expansion method, which describes nonlinear modulated Rossby waves in the geophysical fluid. In addition, he investigated envelope solitary Rossby waves and modulational instability of a uniform Rossby wave train in two space dimensions. Yang et al. $[15,16]$ discussed the effects of periodic external source on the generation of algebraic Rossby solitary waves in stratified fluid and studied the effects of topographic forcing and dissipation on solitary Rossby waves.

In this paper, a dissipative nonlinear Schrödinger equation is derived by employing multiple scales in stratified fluids. In addition, we study the effect of shear of basic flow, Brunt-Vaisala frequency, and $\beta$ effect on inducing envelope 
solitary Rossby waves. Especially, we solve dissipative nonlinear Schrödinger equation by using trial function method and discuss the effects of dissipation on the evolution of envelope solitary Rossby waves.

\section{Governing Equation and Boundary Conditions}

Rossby waves are large-scale atmospheric movement and satisfy the dimensionless quasigeostrophic potential vorticity equation as follows $[17,18]$ :

$$
\begin{aligned}
& \left(\frac{\partial}{\partial t}+\frac{\partial \psi}{\partial x} \frac{\partial}{\partial y}-\frac{\partial \psi}{\partial y} \frac{\partial}{\partial x}\right) \\
& \quad \times\left(\nabla^{2} \psi+\beta(y) y+\frac{f^{2}}{\rho_{s}} \frac{\partial}{\partial z}\left(\frac{\rho_{s}}{N^{2}} \frac{\partial \psi}{\partial z}\right)\right)=0,
\end{aligned}
$$

where $\psi$ is the dimensionless stream function; $\rho_{s}$ is the density; $N(z)$ is called Brunt-Vaisala frequency, which measures the stability of the stratification; $f$ is Coriolis parameter; and $\nabla^{2}$ denotes the two-dimensional Laplace operator and is defined as

$$
\nabla^{2}=\frac{\partial^{2}}{\partial x^{2}}+\frac{\partial^{2}}{\partial y^{2}}
$$

The $\beta$ plane approximation is taken as $\beta(y) ; \beta$ is the function of latitude variable $y$. The lateral boundary conditions are defined as

$$
\frac{\partial \psi}{\partial x}=0, \quad y=0,1
$$

The upper boundary condition satisfies

$$
\rho_{s} \psi \longrightarrow 0, \quad z \longrightarrow \infty .
$$

In lower boundary, considering the existence of turbulent heating dissipation, the governing equation for the thermal equation is

$$
\left(\frac{\partial}{\partial t}+\frac{\partial \psi}{\partial x} \frac{\partial}{\partial y}-\frac{\partial \psi}{\partial y} \frac{\partial}{\partial x}\right) \frac{\partial \psi}{\partial z}+\lambda_{0} \frac{N^{2}}{f} \nabla^{2} \psi=Q, \quad z=0,
$$

where $\lambda_{0}\left(N^{2} / f\right) \nabla^{2} \psi$ means the vorticity dissipation effect induced by Ekman boundary layer, $\lambda_{0}$ is dissipative coefficient, and $Q$ is used to eliminate the dissipation that is caused by shear flow.

\section{Dissipative Nonlinear Schrödinger Equation}

The total stream function is assumed to have the following form:

$$
\psi=-\int^{y}\left(\bar{u}(s, z)-c_{0}\right) d s+\epsilon \bar{\psi}
$$

where $c_{0}$ is a constant and the phase velocity of linear longwave in shear flow; $\epsilon$ is dimensionless Rossby number $\epsilon \ll 1$ and characterizes the strength of the nonlinearity; and $\bar{\psi}$ is disturbed stream function. In order to balance the turbulent dissipation and nonlinearity, we take

$$
\lambda_{0}=\epsilon^{2} \lambda, \quad Q=-\lambda_{0} \frac{N^{2}}{f} \frac{\partial \bar{u}}{\partial y} .
$$

Substitution of (6) and (7) into (1), (5) with the boundary conditions (3), (4) yields

$$
\begin{gathered}
\left(\frac{\partial}{\partial t}+\left(\bar{u}-c_{0}\right) \frac{\partial}{\partial x}+\epsilon\left(\frac{\psi}{\partial x} \frac{\partial}{\partial y}-\frac{\partial \psi}{\partial y} \frac{\partial}{\partial x}\right)\right) \\
\times\left(\nabla^{2} \psi+\frac{f^{2}}{\rho_{s}} \frac{\partial}{\partial z}\left(\frac{\rho_{s}}{N^{2}} \frac{\partial \psi}{\partial z}\right)\right)+\bar{\beta}(y, z) \frac{\partial \psi}{\partial x}=0 \\
\frac{\partial \psi}{\partial x}=0, \quad y=0,1 \\
\left(\frac{\partial}{\partial t}+\left(\bar{u}-c_{0}\right) \frac{\partial}{\partial x}+\epsilon\left(\frac{\psi}{\partial x} \frac{\partial}{\partial y}-\frac{\partial \psi}{\partial y} \frac{\partial}{\partial x}\right)\right) \frac{\partial \psi}{\partial z}-\bar{u}_{z} \frac{\partial \psi}{\partial x} \\
+\lambda \epsilon^{2} \frac{N^{2}}{f} \nabla^{2} \psi=0, \quad z=0,
\end{gathered}
$$

where $\bar{\beta}=\beta-\partial^{2} \bar{u} / \partial y^{2}-\left(f^{2} / \rho_{s}\right)(\partial / \partial z)\left(\left(\rho_{s} / N^{2}\right)(\partial \bar{u} / \partial z)\right)$, and the apostrophe of the $\bar{\psi}$ has been omitted for simplicity.

On account of the existence of these small parameters, we can apply the multiscale expansion method to solve (8)-(11). It is convenient to introduce the following small scales:

$$
T_{1}=\epsilon t, \quad T_{2}=\epsilon^{2} t ; \quad X_{1}=\epsilon x, \quad X_{2}=\epsilon^{2} x,
$$

where $\epsilon$ denotes the weakness of the nonlinearity. The derivative transformations are

$$
\frac{\partial}{\partial t}=\frac{\partial}{\partial t}+\epsilon \frac{\partial}{\partial T_{1}}+\epsilon^{2} \frac{\partial}{\partial T_{2}} ; \quad \frac{\partial}{\partial x}=\frac{\partial}{\partial x}+\epsilon \frac{\partial}{\partial X_{1}}+\epsilon^{2} \frac{\partial}{\partial X_{2}} .
$$

Substitution of (13) into (8), (11) yields

$$
\begin{gathered}
\left(\frac{\partial}{\partial t}+\left(\bar{u}-c_{0}\right) \frac{\partial}{\partial x}\right)\left(\nabla^{2} \psi+\frac{f^{2}}{\rho_{s}} \frac{\partial}{\partial z}\left(\frac{\rho_{s}}{N^{2}} \frac{\partial \psi}{\partial z}\right)\right) \\
+\bar{\beta}(y, z) \frac{\partial \psi}{\partial x} \\
+\epsilon\left\{\left(\frac{\partial \psi}{\partial x} \frac{\partial}{\partial y}-\frac{\partial}{\partial y} \frac{\partial}{\partial x}\right)\left(\nabla^{2} \psi+\frac{f^{2}}{\rho_{s}} \frac{\partial}{\partial z}\left(\frac{\rho_{s}}{N^{2}} \frac{\partial \psi}{\partial z}\right)\right)\right. \\
+\bar{\beta}(y, z) \frac{\partial \psi}{\partial X_{1}}+\left(\frac{\partial}{\partial T_{1}}+\left(\bar{u}-c_{0}\right) \frac{\partial}{\partial X_{1}}\right) \\
\times\left(\nabla^{2} \psi+\frac{f^{2}}{\rho_{s}} \frac{\partial}{\partial z}\left(\frac{\rho_{s}}{N^{2}} \frac{\partial \psi}{\partial z}\right)\right) \\
\left.+2\left(\frac{\partial}{\partial t}+\left(\bar{u}-c_{0}\right) \frac{\partial}{\partial x}\right) \frac{\partial \psi}{\partial x \partial X_{1}}\right\}
\end{gathered}
$$




$$
\begin{gathered}
+\epsilon^{2}\left\{\left(\frac{\partial}{\partial T_{2}}+\left(\bar{u}-c_{0}\right) \frac{\partial}{\partial X_{2}}\right)\left(\nabla^{2} \psi+\frac{f^{2}}{\rho_{s}} \frac{\partial}{\partial z}\left(\frac{\rho_{s}}{N^{2}} \frac{\partial \psi}{\partial z}\right)\right)\right. \\
+2\left(\frac{\partial}{\partial T_{1}}+\left(\bar{u}-c_{0}\right) \frac{\partial}{\partial X_{1}}\right) \frac{\partial \psi}{\partial x \partial X_{1}} \\
+\left(\frac{\partial}{\partial t}+\left(\bar{u}-c_{0}\right) \frac{\partial}{\partial x}\right)\left(\frac{\partial^{2} \psi}{\partial X_{1}^{2}}+2 \frac{\partial^{2} \psi}{\partial x \partial X_{2}}\right) \\
+\bar{\beta}(y, z) \frac{\partial \psi}{\partial X_{2}}+2\left(\frac{\partial \psi}{\partial x} \frac{\partial}{\partial y}-\frac{\partial \psi}{\partial y} \frac{\partial}{\partial x}\right) \frac{\partial^{2} \psi}{\partial x \partial X_{1}} \\
+\left(\frac{\partial \psi}{\partial X_{1}} \frac{\partial}{\partial y}-\frac{\partial \psi}{\partial y} \frac{\partial}{\partial X_{1}}\right) \\
\left.\times\left(\nabla^{2} \psi+\frac{f^{2}}{\rho_{s}} \frac{\partial}{\partial z}\left(\frac{\rho_{s}}{N^{2}} \frac{\partial \psi}{\partial z}\right)\right)\right\}+\epsilon^{3}\{\cdots\}=0 \\
\left.+\frac{\partial}{\partial t}+\left(\bar{u}-c_{0}\right) \frac{\partial}{\partial x}\right) \frac{\partial \psi}{\partial z}-\bar{u}_{z} \frac{\partial \psi}{\partial x} \\
+\epsilon\left\{\left(\frac{\partial \psi}{\partial x} \frac{\partial}{\partial y}-\frac{\partial \psi}{\partial y} \frac{\partial}{\partial x}\right) \frac{\partial \psi}{\partial z}\right. \\
\left.+\left(\frac{\partial}{\partial T_{1}}+\left(\bar{u}-c_{0}\right) \frac{\partial}{\partial X_{1}}\right) \frac{\partial \psi}{\partial z}-\bar{u}_{z} \frac{\partial \psi}{\partial X_{1}}\right\} \\
+\epsilon^{3}\{\cdots\}=0, \quad z=0 . \\
\left.+\epsilon^{2}\left\{\left(\frac{\partial}{\partial T_{2}}+\left(\bar{u}-c_{0}\right) \frac{\partial}{\partial X_{2}}\right) \frac{\partial \psi}{\partial z}-\bar{u}_{z} \frac{\partial \psi}{\partial X_{2}} \frac{\partial \psi}{\partial y} \frac{\partial}{\partial X_{1}}\right) \frac{\partial \psi}{\partial z}+\lambda \frac{N^{2}}{f} \nabla^{2} \psi\right\}
\end{gathered}
$$

Assume the disturbed stream function $\psi$ has the following asymptotic expansion:

$$
\psi=\psi_{0}+\epsilon \psi_{1}+\epsilon^{2} \psi_{2}+\cdots
$$

Substituting (15) into (9), (10), and (14), we can obtain sets of equations and boundary conditions.

To order $O\left(\epsilon^{0}\right)$, we have

$$
\begin{gathered}
\left(\frac{\partial}{\partial t}+\left(\bar{u}-c_{0}\right) \frac{\partial}{\partial x}\right)\left(\nabla^{2} \psi_{0}+\frac{f^{2}}{\rho_{s}} \frac{\partial}{\partial z}\left(\frac{\rho_{s}}{N^{2}} \frac{\partial \psi_{0}}{\partial z}\right)\right) \\
+\bar{\beta}(y, z) \frac{\partial \psi_{0}}{\partial x}=0, \\
\frac{\partial \psi_{0}}{\partial x}=0, \quad y=0,1, \\
\rho_{s} \psi_{0} \longrightarrow 0, \quad z \longrightarrow \infty, \\
\left(\frac{\partial}{\partial t}+\left(\bar{u}-c_{0}\right) \frac{\partial}{\partial x}\right) \frac{\partial \psi_{0}}{\partial z}-\bar{u}_{z} \frac{\partial \psi_{0}}{\partial x}=0, \quad z=0 .
\end{gathered}
$$

Obviously, $\psi_{0}$ satisfies the linear system (16), which has a solution

$$
\psi_{0}=A\left(X_{1}, X_{2} ; T_{1}, T_{2}\right) e^{i(k x-\omega t)} \Phi_{0}(y, z)+\text { c.c },
$$

where c.c is an abbreviation for complex conjugate of its preceding term; $A\left(X_{1}, X_{2}, T_{1}, T_{2}\right)$ is the wave amplitude; $k$ is the zonal wave number; and $\omega$ is the angular frequency.

Substituting (20) into (16)-(19), we can get the following equations for $\Phi_{0}$ :

$$
\begin{gathered}
\left(\frac{d^{2}}{d y^{2}}-k^{2}+\frac{f^{2}}{\rho_{s}} \frac{\partial}{\partial z} \frac{\rho_{s}}{N^{2}} \frac{\partial}{\partial z}+\frac{\bar{\beta}(y, z)}{\bar{u}-c_{0}-c}\right) \Phi_{0}=0, \\
\Phi_{0}(y, z)=0, \quad y=0,1, \\
\rho_{s} \Phi_{0} \longrightarrow 0, \quad z \longrightarrow \infty, \\
\left(\frac{\partial}{\partial z}-\frac{\bar{u}_{z}}{\bar{u}-c_{0}-c}\right) \Phi_{0}(y, z)=0, \quad z=0,
\end{gathered}
$$

where $c=\omega / k$. In the case of order $O\left(\epsilon^{0}\right)$, we can determine the spatial structure of the wave but cannot determine the evolution of the amplitude of the wave with time. In order to determine the evolution of the amplitude $A\left(X_{1}, X_{2}, T_{1}, T_{2}\right)$, we continue to solve higher order of the equation.

For the $O\left(\epsilon^{1}\right)$ problem, we have

$$
\begin{gathered}
\left(\frac{\partial}{\partial t}+\left(\bar{u}-c_{0}\right) \frac{\partial}{\partial x}\right)\left(\nabla^{2} \psi_{1}+\frac{f^{2}}{\rho_{s}} \frac{\partial}{\partial z}\left(\frac{\rho_{s}}{N^{2}} \frac{\partial \psi_{1}}{\partial z}\right)\right) \\
+\bar{\beta}(y, z) \frac{\partial \psi_{1}}{\partial x}=F_{1}, \\
\frac{\partial \psi_{1}}{\partial x}=0, \quad y=0,1, \\
\rho_{s} \psi_{1} \longrightarrow 0, \quad z \longrightarrow \infty, \\
\left(\frac{\partial}{\partial t}+\left(\bar{u}-c_{0}\right) \frac{\partial}{\partial x}\right) \frac{\partial \psi_{1}}{\partial z}-\bar{u}_{z} \frac{\partial \psi_{1}}{\partial x}=E_{1}, \quad z=0
\end{gathered}
$$

where

$$
\begin{aligned}
F_{1}= & \frac{\bar{\beta} \Phi_{0}}{\bar{u}-c_{0}-c}\left(\frac{\partial A}{\partial T_{1}}+c_{1} \frac{\partial A}{\partial X_{1}}\right) e^{i(k x-\omega t)} \\
& +i k\left(\frac{\bar{\beta}}{\bar{u}-c_{0}-c}\right)_{y} A^{2} \Phi_{0}^{2} e^{2 i(k x-\omega t)}+c . c \\
E_{1}= & -\frac{\bar{u}_{z} \Phi_{0}}{\bar{u}-c_{0}-c}\left(\frac{\partial A}{\partial T_{1}}+c \frac{\partial A}{\partial X_{1}}\right) e^{i(k x-\omega t)} \\
& -i k\left(\frac{\bar{u}_{z}}{\bar{u}-c_{0}-c}\right)_{y} A^{2} \Phi_{0}^{2} e^{2 i(k x-\omega t)}+\text { c.c }
\end{aligned}
$$

and $c_{1}=c+2 k^{2}\left(\bar{u}-c_{0}-c\right)^{2} / \bar{\beta},\left(\bar{\beta} /\left(\bar{u}-c_{0}-c\right)\right)_{y}$ and $\left(\bar{u}_{z} /\left(\bar{u}-c_{0}-c\right)\right)_{y}$ denote the first derivative of the variable $y$. 
Using the solvability with $\int_{0}^{\infty} \int_{0}^{1}\left(\Phi_{0} /\left(\bar{u}-c_{0}-c\right)\right) F_{1} d y d z=$ 0 and assuming

$$
\begin{aligned}
& I=\int_{0}^{\infty} \int_{0}^{1} \frac{\bar{\beta} \Phi_{0}^{2}}{\left(\bar{u}-c_{0}-c\right)^{2}} d y d z-\left.\int_{0}^{1} \frac{\bar{u}_{z} \Phi_{0}^{2}}{\left(\bar{u}-c_{0}-c\right)^{2}}\right|_{z=0} d y \\
& I_{1}=\int_{0}^{\infty} \int_{0}^{1} 2 k^{2} \Phi_{0}^{2} d y d z
\end{aligned}
$$

we obtain the following equation:

$$
\frac{\partial A}{\partial T_{1}}+c_{g} \frac{\partial A}{\partial X_{1}}=0
$$

where $c_{g}=c+I_{1} / I$. It is clear from (28) that, in the $O\left(\epsilon^{1}\right)$ solution, the amplitude $A$ propagates at the group velocity $c_{g}$. Then, (22) and (25) can be simplified into

$$
\begin{aligned}
& \left(\frac{\partial}{\partial t}+\left(\bar{u}-c_{0}\right) \frac{\partial}{\partial x}\right)\left(\nabla^{2} \psi_{1}+\frac{f^{2}}{\rho_{s}} \frac{\partial}{\partial z}\left(\frac{\rho_{s}}{N^{2}} \frac{\partial \psi_{1}}{\partial z}\right)\right) \\
& +\bar{\beta}(y, z) \frac{\partial \psi_{1}}{\partial x}=i k\left(\frac{\bar{\beta}}{\bar{u}-c_{0}-c}\right)_{y} A^{2} \Phi_{0}^{2} e^{2 i(k x-\omega t)}+c . c, \\
& \left(\frac{\partial}{\partial t}+\left(\bar{u}-c_{0}\right) \frac{\partial}{\partial x}\right) \frac{\partial \psi_{1}}{\partial z}-\bar{u}_{z} \frac{\partial \psi_{1}}{\partial x} \\
& =-i k\left(\frac{\bar{u}_{z}}{\bar{u}-c_{0}-c}\right)_{y} A^{2} \Phi_{0}^{2} e^{2 i(k x-\omega t)}+\text { c.c, } \quad z=0
\end{aligned}
$$

We assume that $\psi_{1}$ has the following wave-like form:

$$
\psi_{1}=B\left(X_{1}, X_{2} ; T_{1}, T_{2}\right) e^{2 i(k x-\omega t)} \Phi_{1}(y)+\text { c.c } ;
$$

substituting (30) into (29) yields

$$
\begin{gathered}
B\left(\frac{d^{2}}{d y^{2}}-4 k^{2}+\frac{f^{2}}{\rho_{s}} \frac{\partial}{\partial z} \frac{\rho_{s}}{N^{2}} \frac{\partial}{\partial z}+\frac{\bar{\beta}}{\bar{u}-c_{0}-c}\right) \Phi_{1}(y, z) \\
=A^{2} G_{1}(y, z), \\
G_{1}(y, z)=\left(\frac{\bar{\beta}}{\bar{u}-c_{0}-c}\right)_{y} \frac{\Phi_{0}^{2}}{2\left(\bar{u}-c_{0}-c\right)}, \\
B\left(\frac{\partial}{\partial z}-\frac{\bar{u}_{z}}{\bar{u}-c_{0}-c}\right) \Phi_{1}(y, z)=A^{2} G_{2}(y, z), \\
G_{2}(y, z)=\left(\frac{\bar{u}_{z}}{\bar{u}-c_{0}-c}\right)_{y} \frac{\Phi_{0}^{2}}{2\left(\bar{u}-c_{0}-c\right)} .
\end{gathered}
$$

Obviously, $A, B$ are two dependent variables, and $B$ is related to $A^{2}$ in (31). We assume that $B=A^{2}$ for simplicity; then,

$$
\psi_{1}=A^{2}\left(X_{1}, X_{2} ; T_{1}, T_{2}\right) e^{2 i(k x-\omega t)} \Phi_{1}(y)+\text { c.c. }
$$

To order $O\left(\epsilon^{2}\right)$, we have

$$
\begin{gathered}
\left(\frac{\partial}{\partial t}+\left(\bar{u}-c_{0}\right) \frac{\partial}{\partial x}\right)\left(\nabla^{2} \psi_{2}+\frac{f^{2}}{\rho_{s}} \frac{\partial}{\partial z}\left(\frac{\rho_{s}}{N^{2}} \frac{\partial \psi_{2}}{\partial z}\right)\right) \\
+\bar{\beta}(y, z) \frac{\partial \psi_{2}}{\partial x}=F_{2}, \\
\frac{\partial \psi_{2}}{\partial x}=0, \quad y=0,1, \\
\rho_{s} \psi_{2} \rightarrow 0, \quad z \longrightarrow \infty, \\
\left(\frac{\partial}{\partial t}+\left(\bar{u}-c_{0}\right) \frac{\partial}{\partial x}\right) \frac{\partial \psi_{2}}{\partial z}-\bar{u}_{z} \frac{\partial \psi_{2}}{\partial x}=E_{2}, \quad z=0
\end{gathered}
$$

where

$$
\begin{aligned}
F_{2}= & \frac{\bar{\beta}}{\bar{u}-c_{0}-c} \Phi_{0}\left(\frac{\partial A}{\partial T_{2}}+c_{1} \frac{\partial A}{\partial X_{2}}\right) e^{i(k x-\omega t)} \\
& +i k\left(2 c_{g}+c+3 c_{0}-3 \bar{u}\right) \Phi_{0} \frac{\partial^{2} A}{\partial X_{1}^{2}} e^{i(k x-\omega t)} \\
& +i k|A|^{2} A\left\{\Phi_{0} \frac{\partial G_{1}}{\partial y}+\Phi_{0} \Phi_{1}\left(\frac{\bar{\beta}}{\bar{u}-c_{0}-c}\right)_{y}\right. \\
E_{2}= & -\frac{\bar{u}_{z} \Phi_{0}}{\bar{u}-c_{0}-c}\left(\frac{\partial A}{\partial T_{2}}+c \frac{\partial A}{\partial X_{2}}\right) e^{i(k x-\omega t)} \\
& -i k|A|^{2} A\left(\Phi_{0} \frac{\partial G_{2}}{\partial y}-\Phi_{0} \frac{\partial \Phi_{0}}{\partial y}\right\} e^{i(k x-\omega t)}+c \cdot c+\square, \\
& \left.+\lambda \frac{\bar{u}_{z}}{f}\right)_{y} \\
& \left.+c . c+\square, \quad \frac{c_{0}-c}{\bar{u}-c_{0}-c}+\frac{f^{2}}{\rho_{s}} \frac{\partial}{\partial z} \frac{\rho_{s}}{N^{2}} \frac{\partial \Phi_{0}}{\partial z}\right)_{0} A e^{i(k x-\omega t)} \\
& +2 G_{2} \frac{\partial y}{i(k x-\omega t)}
\end{aligned}
$$

where $\square$ expresses the terms associated with $e^{ \pm 2 i(k x-\omega t)}$, $e^{ \pm 3 i(k x-\omega t)}$, and so on.

Similarly, using the solvability conditions associated with (34), we can obtain the amplitude $A$ satisfying the following NLS equation with a dissipation item:

$$
i\left(\frac{\partial A}{\partial T_{2}}+c_{g} \frac{\partial A}{\partial X_{2}}\right)+\alpha \frac{\partial^{2} A}{\partial X_{1}^{2}}+\delta|A|^{2} A+i \gamma A=0
$$


where

$$
\begin{aligned}
& \alpha=-\frac{I_{2}}{I}, \quad \delta=-\frac{I_{3}}{I}, \quad \gamma=\frac{I_{4}}{I}, \\
& I_{2}=k \int_{0}^{\infty} \int_{0}^{1} \frac{2 c_{g}+c+3 c_{0}-3 \bar{u}}{\bar{u}-c_{0}-c} \Phi_{0}^{2} d y d z \\
& I_{3}=k \int_{0}^{\infty} \int_{0}^{1} \frac{\Phi_{0}}{\left(\bar{u}-c_{0}-c\right)}\left\{\Phi_{0} \frac{\partial G_{1}}{\partial y}+\Phi_{0} \Phi_{1}\left(\frac{\bar{\beta}}{\bar{u}-c_{0}-c}\right)_{y}\right. \\
& \left.-k \int_{0}^{1} \frac{\Phi_{0}}{\left(\bar{u}-c_{0}-c\right)}\left(\Phi_{0} \frac{\partial G_{2}}{\partial y}-\Phi_{0} \frac{\partial \Phi_{0}}{\partial y}\right\} d y d z, \overline{\bar{u}-c_{0}-c}\right)_{y} \\
& \left.I_{4}=\lambda \int_{0}^{1} \frac{N^{2} \Phi_{0}}{f\left(\bar{u}-c_{0}-c\right)} \quad+2 G_{2} \frac{\partial \Phi_{0}}{\partial y}\right)\left.\right|_{z=0} d y, \\
& \times\left.\left(\frac{\bar{\beta} \Phi_{0}}{\bar{u}-c_{0}-c}+\frac{f^{2}}{\rho_{s}} \frac{\partial}{\partial z} \frac{\rho_{s}}{N^{2}} \frac{\partial \Phi_{0}}{\partial z}\right)\right|_{z=0} d y .
\end{aligned}
$$

We called (35) dissipative nonlinear Schrödinger equation (DNLSE); the coefficients $\alpha, \delta$ are the so-called dispersion and landau coefficients, respectively, which are related to $\bar{\beta}(y, z)$ and $\bar{u}(y, z)$.

DNLSE (35) describes the evolution of envelop solitary Rossby waves amplitude under the dissipation and $\beta$ effect and reflects the characteristics of Rossby waves. If we introduce the following coordinates transformation defined by Jeffrey and Kawahara [19],

$$
T=T_{2}, \quad X=\frac{1}{\epsilon}\left(X_{2}-c_{g} T_{2}\right)=X_{1}-c_{g} T_{1},
$$

then DNLSE (35) can be rewritten as

$$
i \frac{\partial A}{\partial T}+\alpha \frac{\partial^{2} A}{\partial X^{2}}+\delta|A|^{2} A+i \gamma A=0 .
$$

If there is no shear in the basic flow $(\bar{u}=$ constant $)$ and $\beta$ is the function of latitudinal variable $y$, then $\delta \neq 0$; (38) is still the nonlinear Schrödinger equation. That is to say, though there is no shear in the basic flow, the evolution of envelope solitary Rossby waves also satisfies the nonlinear Schrödinger equation as long as the $\beta$ effects exist. Only when the basic flow has no shear and $\beta$ is a constant, the nonlinear term of (38) will disappear. We are also aware that the condition

$$
\begin{aligned}
I= & \int_{0}^{\infty} \int_{0}^{1} \frac{\bar{\beta} \Phi_{0}^{2}}{\left(\bar{u}-c_{0}-c\right)^{2}} d y d z \\
& -\left.\int_{0}^{1} \frac{\bar{u}_{z} \Phi_{0}^{2}}{\left(\bar{u}-c_{0}-c\right)^{2}}\right|_{z=0} d y \neq 0
\end{aligned}
$$

is also essential to the existence of envelope solitary waves. This means that it cannot produce barotropic instability. In fact, once the barotropic instability is produced, it is impossible for the envelope solitary wave to maintain a constant waveform.

In the absence of dissipation $(\gamma=0)$, (38) can be transformed into the following standard NLSE:

$$
i \frac{\partial A}{\partial T}+\alpha \frac{\partial^{2} A}{\partial X^{2}}+\delta|A|^{2} A=0 .
$$

The single envelope soliton solution of $(40)$ is

$$
\begin{aligned}
A(X, T)= & \sqrt{\frac{2 \alpha}{\delta}} A_{0} \operatorname{sech}\left(A_{0}\left(X-2 \alpha k_{0} T\right)\right) \\
& \times e^{i\left(k_{0} X-\alpha\left(k_{0}^{2}-A_{0}^{2}\right) T\right)},
\end{aligned}
$$

where $A_{0}$ and $k_{0}$ denote the amplitude and moving speed of envelope solitary Rossby waves, whose value is determined by the initial state of $A(X, T)$. A very notable feature of envelope solitary Rossby waves is that the speed is independent of amplitude, but the carrier wave is related to amplitude. The computer simulation of the solution (41) is presented in Figure 1 . In the absence of nonlinear term $(\delta=0),(40)$ can be transformed into the following form:

$$
i \frac{\partial A}{\partial T}+\alpha \frac{\partial^{2} A}{\partial X^{2}}=0
$$

In order to discuss the nonlinear effect, the initial condition is taken as follows:

$$
A(X, 0)=A_{0} \operatorname{sech}\left(A_{0} X\right) \times e^{i k_{0} X},
$$

and the simulated result of (42) is shown in Figure 2. From Figure 2, we can find that the envelope solitary wave cannot maintain its initial waveform, and the amplitude of envelope solitary Rossby waves decreases and the width of envelope solitary Rossby waves increases with time.

Substitution of (41) and (15) into (6) yields

$$
\begin{aligned}
\psi= & -\int^{y}\left(\bar{u}(s, z)-c_{0}\right) d s \\
& +\epsilon \sqrt{\frac{2 \alpha}{\delta}} A_{0} \operatorname{sech}\left(\epsilon A_{0}(x-V t)\right) \times \Phi_{0} e^{i(K x-\Omega t)},
\end{aligned}
$$

where

$$
\begin{aligned}
& V=c_{g}+2 \epsilon \alpha k_{0}, \quad K=k+\epsilon k_{0}, \\
& \Omega=\omega+\epsilon k_{0} c_{g}+\epsilon^{2} \alpha\left(k_{0}^{2}-A_{0}^{2}\right) .
\end{aligned}
$$

Equations (44) and (45) show that the envelope soliton propagation velocity $V$ is equal to the group velocity of Rossby waves plus a small correction; the carrier wave number $k$ is equal to the number of linear Rossby waves plus a small correction; the carrier frequency $\Omega$ is equal to the linear Rossby waves frequency $\omega$ plus two small corrections, related to its amplitude. That reveals the nonlinear characteristics. 

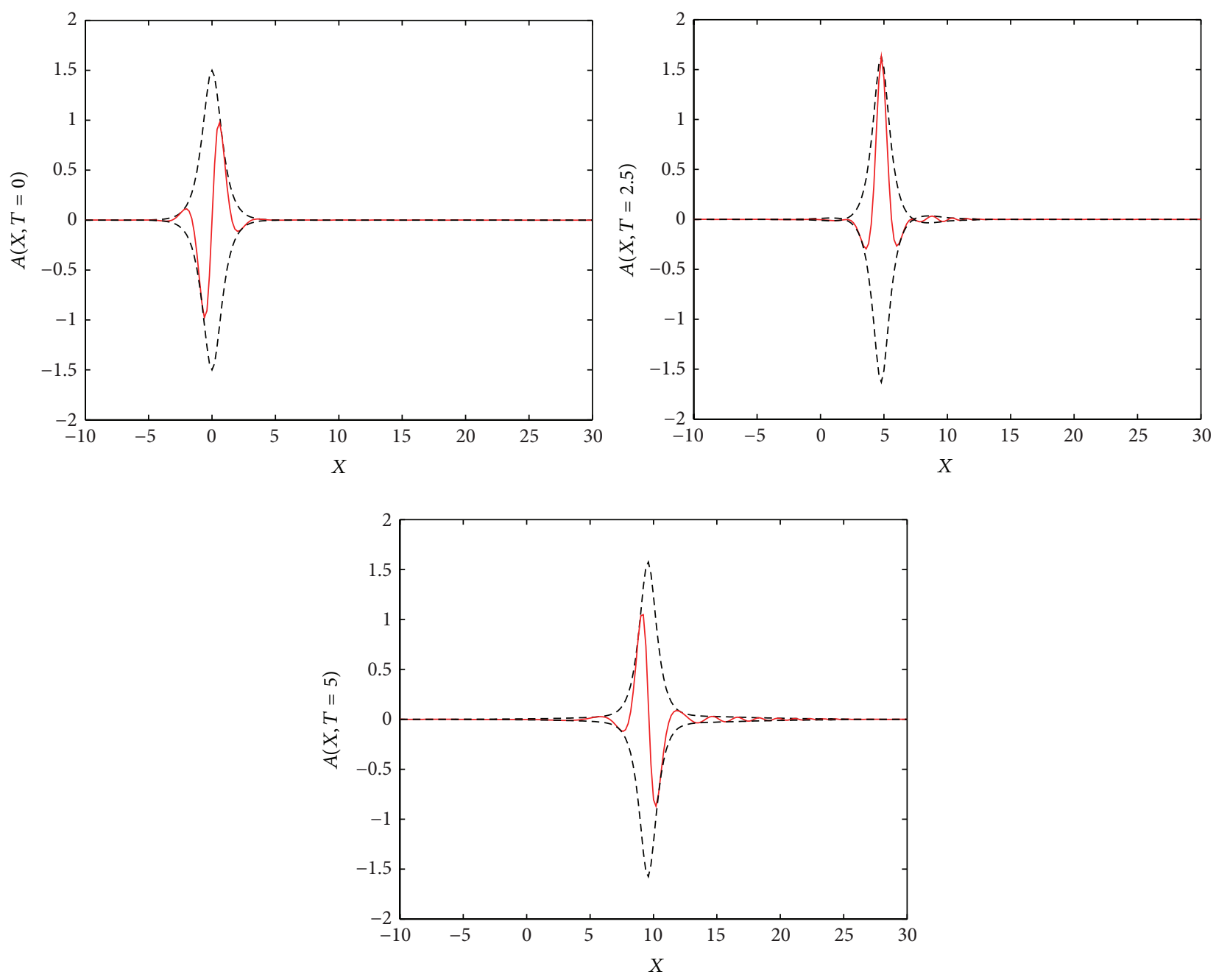

Figure 1: $\alpha=1 / 2, \delta=1, A_{0}=3 / 2, k_{0}=2$.

\section{Dissipation Effect on the Evolution of Rossby Envelope Solitary Waves}

In this section, we will analyze the dissipation effects on the evolution of envelope solitary Rossby waves based on the asymptotic solution. For the DNLSE $(\gamma \ll 1)$,

$$
i \frac{\partial A}{\partial T}+\alpha \frac{\partial^{2} A}{\partial X^{2}}+\delta|A|^{2} A+i \gamma A=0
$$

assuming the solution of (46) has the following form:

$$
\begin{aligned}
A= & \sqrt{\frac{2 \alpha}{\delta}} C(T) \operatorname{sech}(B(T)(X-v(T))) \\
& \times \exp (i(k(T) X-w(T))) .
\end{aligned}
$$

Substituting (47) into (46), we can get the following equation:

$$
\begin{aligned}
& \sqrt{\frac{2 \alpha}{\delta}} \frac{C(T)}{\cosh (B(T)(X-v(T)))} \\
& \times\left(-\frac{d k(T)}{d T} X+\frac{d w(T)}{d T}-\alpha\left(k^{2}(T)-B^{2}(T)\right)\right) \\
& +2 \alpha \sqrt{\frac{2 \alpha}{\delta}} \frac{C(T)}{\cosh ^{3}(B(T)(X-v(T)))}\left(C^{2}(T)-B^{2}(T)\right) \\
& +i \sqrt{\frac{2 \alpha}{\delta}}\left\{-\frac{C(T) \sinh (B(T)(X-v(T)))}{\cosh ^{2}(B(t)(X-v(T)))}\right. \\
& \times\left(\frac{d B(T)}{d T} X-\frac{d B(T)}{d T} v(T)\right. \\
& \left.-B(T) \frac{d v(T)}{d T}+2 \alpha B(T) k(T)\right)
\end{aligned}
$$



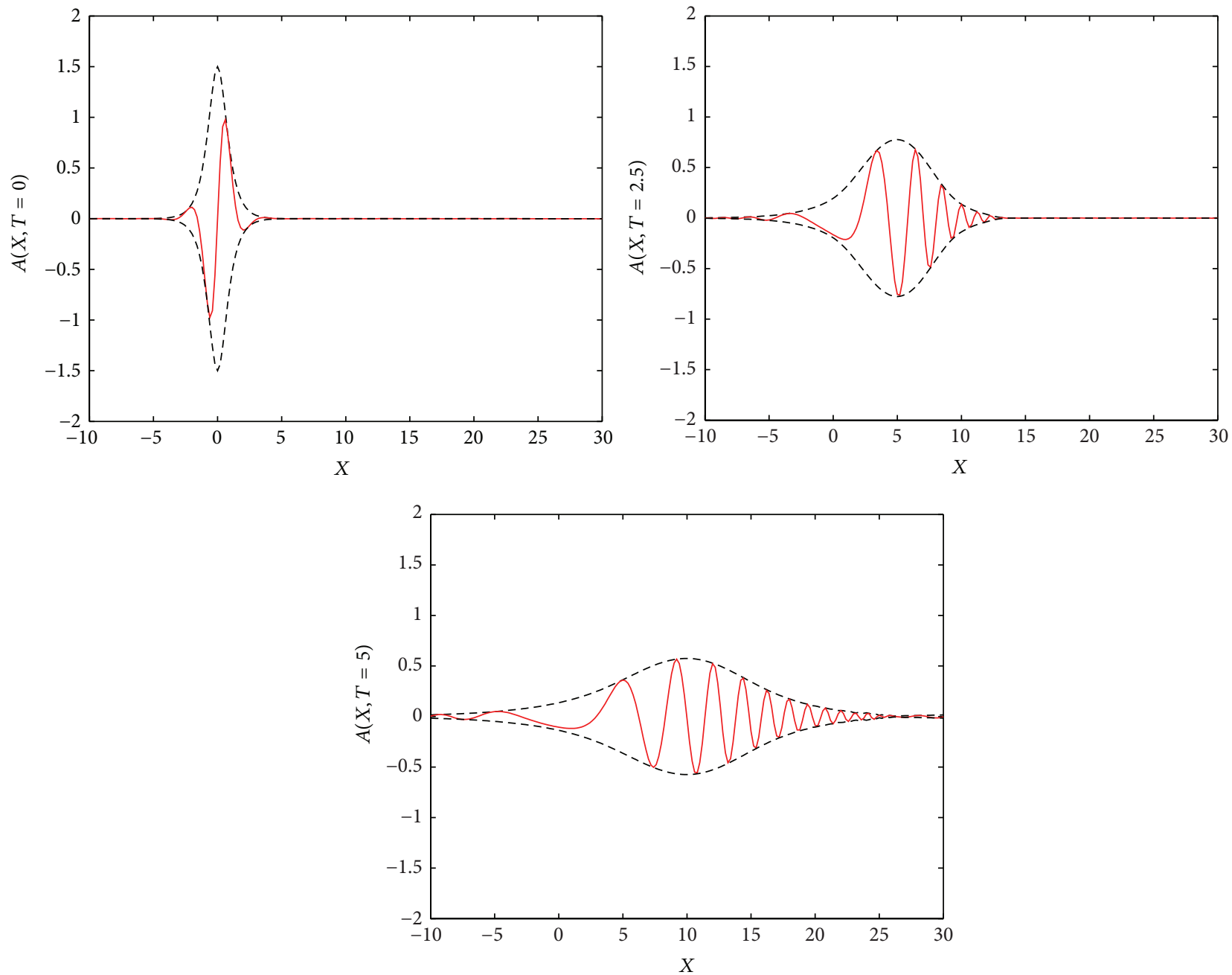

Figure $2: \alpha=1 / 2, \delta=0, A_{0}=3 / 2, k_{0}=2$.

$$
\begin{aligned}
& +\frac{1}{\cosh (B(T)(X-v(T)))} \\
& \left.\times\left(\frac{d C(T)}{d T}+\gamma C(T)\right)\right\}=0 .
\end{aligned}
$$

Conditions on the $B(T), C(T), k(T), \omega(T), v(T)$ are determined by equating coefficients of $\cosh ^{n}(B(T)(X-v(T)))$, $(n \in \mathscr{Z})$ by zero. This will produce a series of PDEs as follows:

$$
\begin{gathered}
\frac{d C(T)}{d T}+\gamma C(T)=0, \\
C^{2}(T)-B^{2}(T)=0, \\
\frac{d \omega(T)}{d T}-\alpha\left(k^{2}(T)-B^{2}(T)\right)-\frac{d k(T)}{d T} X=0, \\
\frac{d B(T)}{d T}(X-v(T))-B(T)\left(\frac{d v(T)}{d T}-2 \alpha k(T)\right)=0 .
\end{gathered}
$$

From (49) and (50), we can get

$$
C(T)=B(T)=h e^{-\gamma T} .
$$

From (51), we can get

$$
\frac{d k(T)}{d T}=0, \quad \frac{d \omega(T)}{d T}-\alpha\left(k^{2}(T)-B^{2}(T)\right)=0,
$$

and the solution of (54) is

$$
k(T)=k_{0}, \quad \omega(T)=\alpha\left(k_{0}^{2} T+\frac{h^{2}}{2 \gamma} e^{-2 \gamma T}\right) .
$$

Substituting (53) into (52), we obtain

$$
\gamma(X-v(T))+\left(\frac{d v(T)}{d T}-2 \alpha k(T)\right)=0 .
$$

Because of $\gamma \ll 1$, the term of $\gamma(X-v(T))$ can be omitted in (56). We can get

$$
v(T)=\int 2 \alpha k_{0} d T=2 \alpha k_{0} T
$$




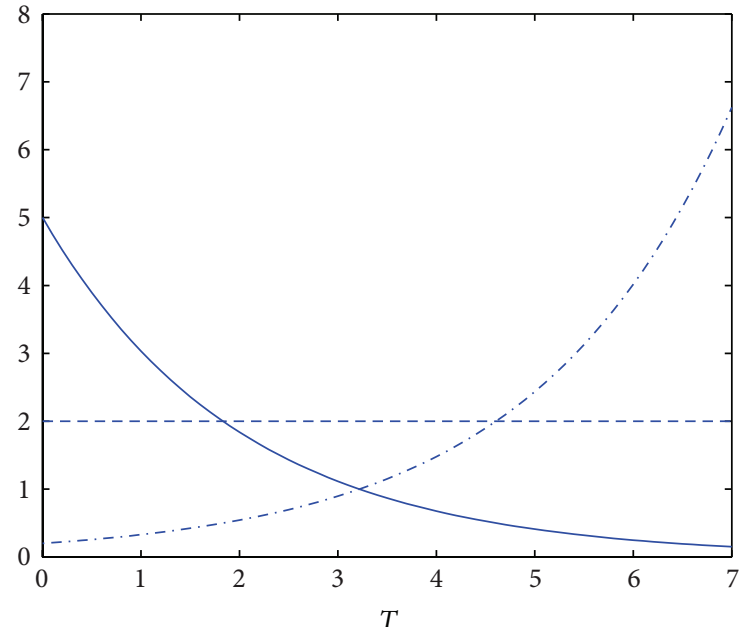

$$
\begin{aligned}
& \text { _ Amplitude } \\
& \text { - - Velocity } \\
& \text {-. - Width }
\end{aligned}
$$

FIGURE 3: Variation of amplitude, velocity, and width due to the presence of dissipation effect.

By virtue of (53), (55), and (57), the asymptotic solution of the DNLSE (46) is

$$
\begin{aligned}
A= & \sqrt{\frac{2 \alpha}{\delta}} h e^{-\gamma T} \operatorname{sech}\left\{h e^{-\gamma T}\left(X-2 \alpha k_{0} T\right)\right\} \\
& \times e^{i\left(k_{0} X-\alpha\left(k_{0}^{2} T+\left(h^{2} / 2 \gamma\right) e^{-2 \gamma T}\right)\right)} .
\end{aligned}
$$

Figure 3 describes the variation of amplitude, velocity, and width due to the presence of dissipation effect, and we can get the following conclusions. The amplitude of wave is decreasing with time as $h e^{-\gamma T}$, where $h$ is the initial amplitude. As the amplitude decreases with time, the width of the wave $1 / h e^{\gamma T}$ becomes wider. However, dissipation has no effect on the propagation velocity of envelope solitary wave, and this is quite different from the solitary wave of KdV-type [20-22]. This conclusion can also be obtained by Tan [9], but the analytical solution of DNLSE was not given in that paper. For the case of KdV-type solitary wave, dissipation not only weakens the strength of solitary wave but also slows the propagation velocity down. It is notable that dissipation has certain influence on the carrier frequency.

Substituting (58) and (15) into (6), we obtain

$$
\begin{aligned}
\psi= & -\int^{y}\left(\bar{u}(s, z)-c_{0}\right) d s \\
& +\epsilon \sqrt{\frac{2 \alpha}{\delta}} h e^{-\gamma \epsilon^{2} t} \operatorname{sech}\left\{\epsilon h e^{-\gamma \epsilon^{2} t}\left(X-V^{\prime} t\right)\right\} \times \Phi_{0} e^{i\left(K^{\prime} X-\Omega^{\prime}\right)},
\end{aligned}
$$

where

$$
\begin{aligned}
& V^{\prime}=c_{g}+2 \epsilon \alpha k_{0}, \quad K^{\prime}=k+\epsilon k_{0}, \\
& \Omega^{\prime}=\left(\omega+\epsilon k_{0} c_{g}+\epsilon^{2} \alpha k_{0}^{2}\right) t+\frac{\alpha h^{2}}{2 \gamma} e^{-2 \gamma \epsilon^{2} t} .
\end{aligned}
$$

If we let $\gamma \rightarrow 0$, (58), (59), and (60) reduce to (41), (44), and (45), respectively.

\section{Conclusions}

In the paper, we derive the so-called DNLSE by means of multiple scales analysis and perturbation method to describe envelope solitary Rossby waves with dissipation effect in stratified fluids. By analyzing the evolution of amplitude of envelope solitary Rossby waves, we can find that the shear of basic flow, Brunt-Vaisala frequency, and $\beta$ effect are important factors to form the envelope solitary Rossby waves. In addition, we get the asymptotic solution of DNLSE by employing trial function method. With the help of the asymptotic solution, the effect of dissipation on the evolution of envelope solitary Rossby waves is thoroughly investigated. The results show that the dissipation causes slow decrease of amplitude of the envelope solitary Rossby waves and slow increase of width, while it has no effects on the propagation velocity. That is quite different from the KdV-type solitary waves. It is notable that dissipation has certain influence on the carrier frequency. It is also interesting for us to see how the governing equation of envelope solitary Rossby waves will be in the rotating stratified fluids. In the forthcoming days, we will further discuss these problems.

\section{Conflict of Interests}

The authors declare that there is no conflict of interests regarding the publication of this paper.

\section{Acknowledgments}

This research work has been supported financially by Strategic Pioneering Program of Chinese Academy of Sciences (no. XDA10020104), Global Change and Air-Sea Interaction (no. GASI-03-01-01-02), National Natural Science Foundation of China through Grants (41376030, 41376029, and 41106017), Nature Science Foundation of Shandong Province of China (no. ZR2013AQ017), Science and Technology Plan Project of Qingdao (no. 14-2-4-77-jch), Open Fund of the Key Laboratory of Data Analysis and Application, and State Oceanic Administration (no. LDAA-2013-04).

\section{References}

[1] R. R. Long, "Solitary waves in the westerlies," Journal of the Atmospheric Sciences, vol. 21, no. 2, pp. 197-200, 1964.

[2] D. J. Benney, "Long non-linear waves in fluid flows," Journal of Mathematical Physics, vol. 45, pp. 52-63, 1966.

[3] P. E. Holloway, E. Pelinovsky, and T. Talipova, "A generalized Korteweg-de Vries model of internal tide transformation in the coastal zone," Journal of Geophysical Research C: Oceans, vol. 104, no. 8, pp. 18333-18350, 1999.

[4] Z. H. Xu, B. S. Yin, Y. J. Hou, and A. K. Liu, "Seasonal variability and north õ south asymmetry of internal tides in the deep basin west of the Luzon Strait," Journal of Marine Systems, vol. 134, pp. 101-112, 2014. 
[5] Z. Xu, B. Yin, Y. Hou, and Y. Xu, "Variability of internal tides and near-inertial waves on the continental slope of the northwestern South China Sea," Journal of Geophysical Research: Oceans, vol. 118, no. 1, pp. 197-211, 2013.

[6] A. Domaracki and A. Z. Loesh, "Nonlinear interactions among equatorial waves," Journal of the Atmospheric Sciences, vol. 34, no. 3, pp. 486-498, 1977.

[7] P. Ripa, "Variability of the tropical Atlantic Ocean," Journal of Physical Oceanography, vol. 12, article 97, 1982.

[8] L. G. Yang, C. J. Da, J. Song, H. Zhang, H. Yang, and Y. Hou, "Rossby waves with linear topography in barotropic fluids," Chinese Journal of Oceanology and Limnology, vol. 26, no. 3, pp. 334-338, 2008.

[9] B. K. Tan, "Envelope solitary Rossby waves and their interactions under the inuence of forcing and dissipation," Scientia Atmospherica Sinica, p. 03, 1995.

[10] J. Song and L. Yang, "Force solitary Rossby waves with beta effect and topography effect in stratified flows," Acta Physica Sinica, vol. 59, no. 5, pp. 3309-3314, 2010.

[11] J. Song and L. G. Yang, "Nonlinear Rossby waves excited slowly changing underlying surface and dissipation," Acta Physica Sinica, vol. 63, no. 3, Article ID 060401, 2014.

[12] S. A. Maslowe and L. G. Redekopp, "Long nonlinear waves in stratified shear flows," Journal of Fluid Mechanics, vol. 101, no. 2, pp. 321-348, 1980.

[13] D. Luo, "Derivation of a higher order nonlinear Schrödinger equation for weakly nonlinear Rossby waves," Wave Motion, vol. 33, no. 4, pp. 339-347, 2001.

[14] D. H. Luo, "Envelope solitary Rossby waves and modulational instabilities of uniform Rossby wave trains in two space dimensions," Wave Motion, vol. 24, no. 3, pp. 315-325, 1996.

[15] H. W. Yang, X. R. Wang, and B. S. Yin, "A kind of new algebraic Rossby solitary waves generated by periodic external source," Nonlinear Dynamics, vol. 76, no. 3, pp. 1725-1735, 2014.

[16] H. W. Yang, B. S. Yin, and Y. L. Shi, "Forced dissipative Boussinesq equation for solitary waves excited by unstable topography," Nonlinear Dynamics, vol. 70, no. 2, pp. 1389-1396, 2012.

[17] B. Wang and H. Y. Weng, Introduction of Geophysical Fluid Dynamics, Ocean Press, Beijing, China, 1981.

[18] S. K. Liu and S. D. Liu, Atmospheric Dynamics, Beijing University Press, Beijing, China, 1991.

[19] A. Jeffrey and T. Kawahara, Asymptotic Methods in Nonlinear Wave Theory, Pitman, London, UK, 1982.

[20] P. Malanotte Rizzoli, "Planetary solitary waves in geophysical flows," Advances in Geophysics, vol. 24, pp. 147-224, 1982.

[21] J. C. McWilliams, G. R. Flierl, V. D. Larichev, and G. M. Reznik, "Numerical studies of barotropic modons," Dynamics of Atmospheres and Oceans, vol. 5, no. 4, pp. 219-238, 1981.

[22] M. J. Ablowitz and H. Segur, Solitons and the Inverse Scattering Transform, SIAM, Philadelphia, Pa, USA, 1981. 


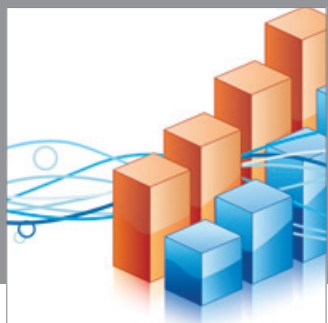

Advances in

Operations Research

mansans

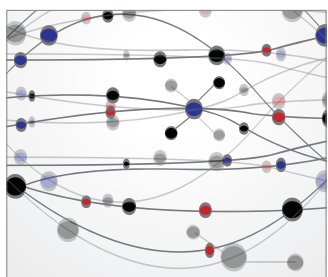

The Scientific World Journal
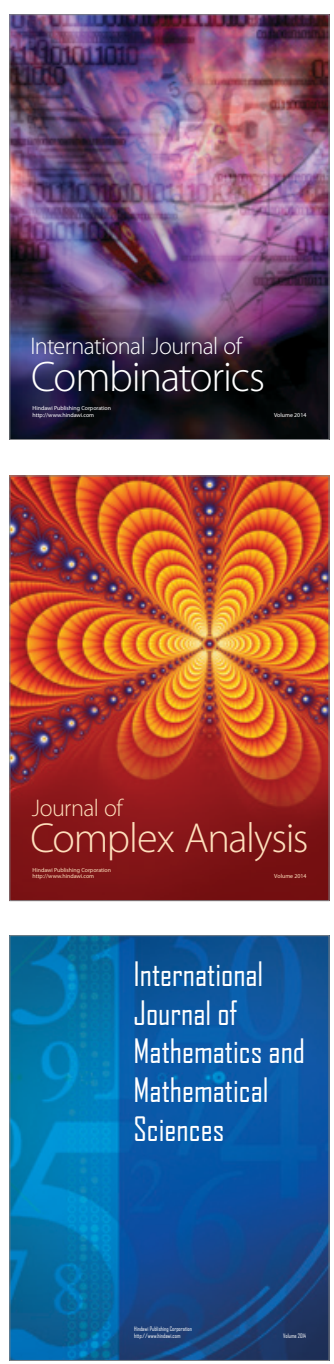
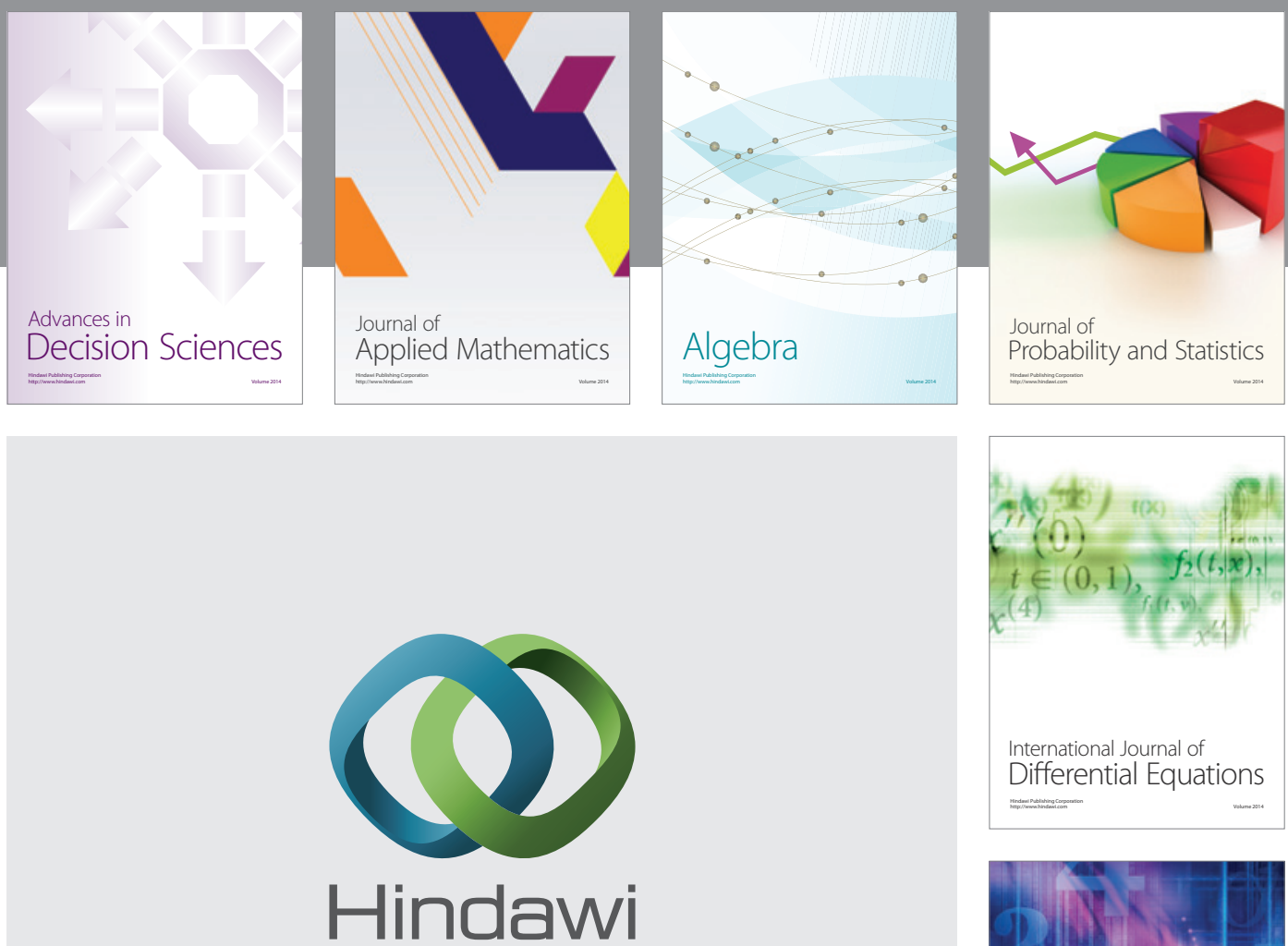

Submit your manuscripts at http://www.hindawi.com
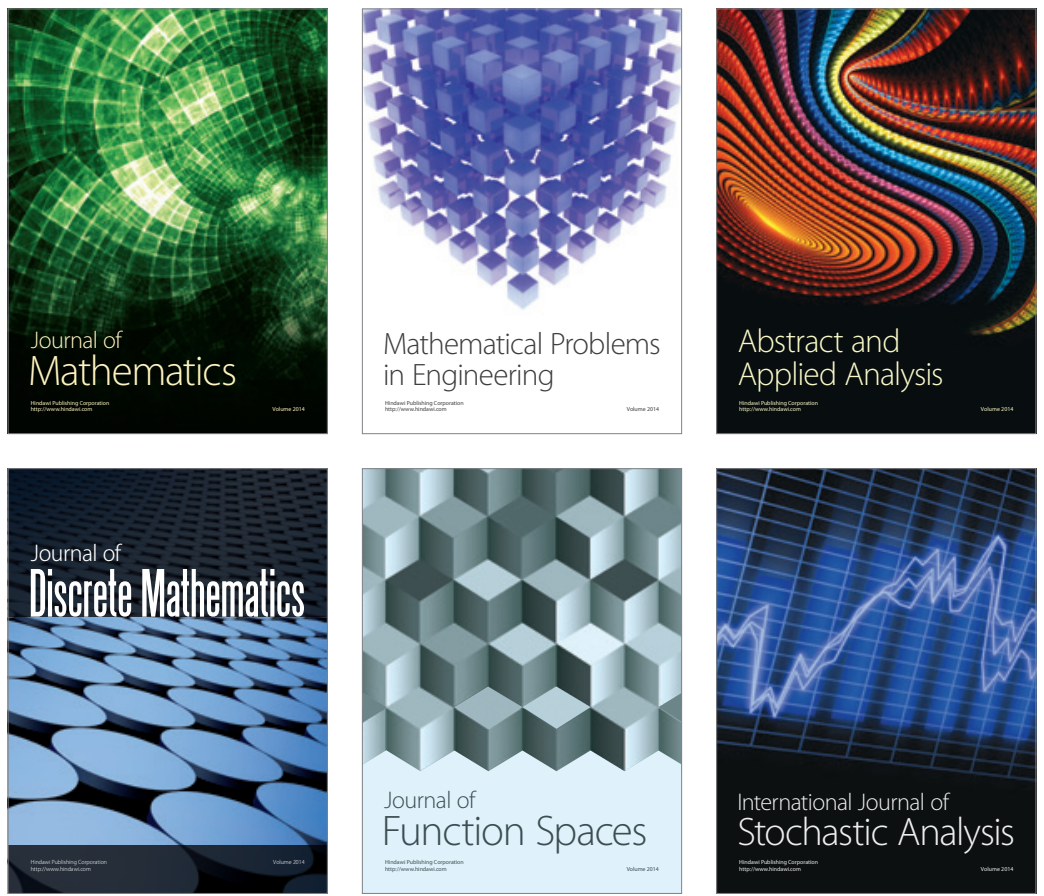

Journal of

Function Spaces

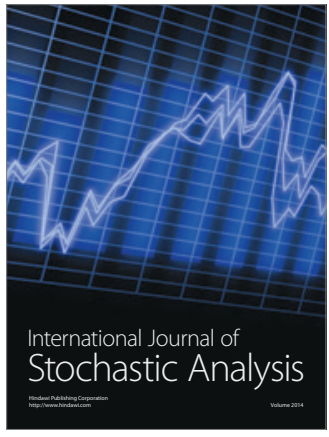

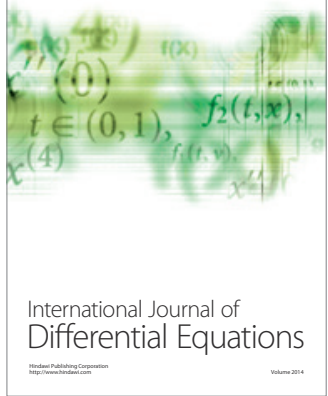
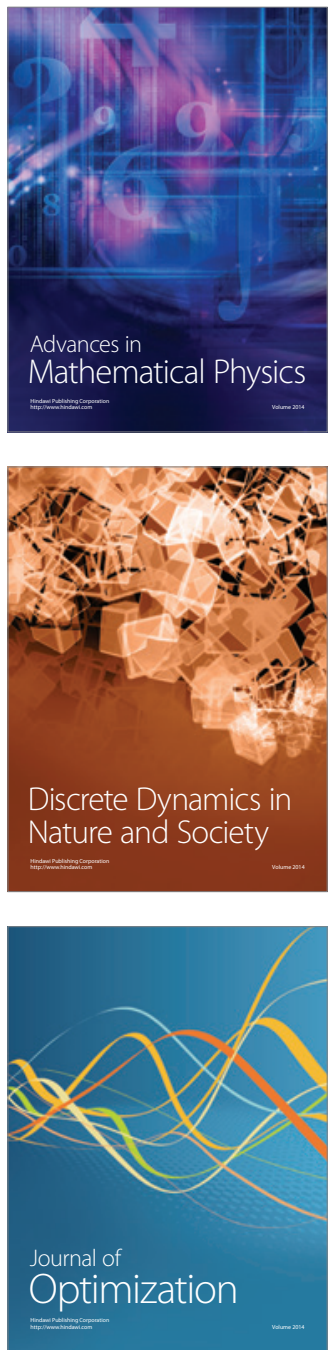